\title{
The Local Conformation of Poly(dimethylsiloxane)
}

\author{
Geoffrey R. Mitchell, ${ }^{\dagger}$ and Akira Odajima* \\ Department of Metallurgy and Materials Science, \\ University of Cambridge, Pembroke Street, \\ Cambridge, CB2 3QZ, U.K. \\ *Department of Applied Physics, Faculty of Engineering, \\ Hokkaido University, Sapporo 060, Japan
}

(Received February 15, 1984)

\begin{abstract}
Wide-angle X-ray scattering (WAXS) data obtained at room temperature from bulk poly(dimethylsiloxane) is presented and compared with the scattering functions calculated for random single chains in which the skeletal bond rotation angles have been assigned according to the precepts of the rotational isomeric state (RIS) approximation. There are significant differences between experimental and calculated curves, which are not eliminated by the introduction of large thermal-like fluctuations of the bond rotation angles. It is suggested that the rotation states are delocalised in PDMS, as the scattering calculated for a chain with free rotation of the skeletal bonds provides a more satisfactory fit to the experimental data. The relevance of the RIS approach for describing the local conformation of polymer molecules is discussed and the need to sample the spatial distribution function of the chain at different scales is stressed.

KEY WORDS Poly(dimethylsiloxane) / Conformational Analysis / X-Ray

Scattering / Rotational Isomeric State Approximation /
\end{abstract}

The characteristics of the trajectories of individual polymer molecules have been extensively studied, both theoretically and by experiment. ${ }^{1}$ In these studies the use of the rotational isomeric state (RIS) model $^{2}$ has been particularly successful in providing a framework within which a broad understanding of the configuration dependent properties of macromolecules has developed. However, recently the approximations inherent in the RIS approach have been critically examined. ${ }^{3-11}$ Procedures have been presented which allow for the inclusion of thermal-like fluctuations in the values assigned to the skeletal bond rotations angles, ${ }^{3,4,10,11}$ and more radical approaches have been considered which evaluate the chain properties taking account of all possible molecular configurations. ${ }^{5,9}$ The result of each of these studies suggests that many of the discrepancies and anomalies observed in previous studies may be accounted for by abandoning the approximations of the RIS model for a more exact treatment. A common feature of configurational studies is that the correctness of the proposed model is evaluated by comparing experimental measurements of the mean square end-to-end vector or similar large correlation distance functions with those calculated on the basis of the model. Thus while the properties measured are dependent upon longrange correlations, the input to the models, whether it be a RIS or modified model, is essentially of a short range nature, defining the interdependence of the conformational energy for skeletal bond rotations. Such interdependence is usually restricted to first and second nearest neighbour bonds. Under these circumstances it would seem more direct to attempt to determine the characteristics of the local

\footnotetext{
${ }^{\dagger}$ Present address: J. J. Thomson Laboratory, University of Reading, Whiteknights, Reading, RG6 2AF, U.K.
} 
conformation, before extending the model to large scale correlations. For that situation, wide-angle X-ray scattering (WAXS) techniques which are sensitive to correlations in the range 1 to $20 \AA^{12}$ appear particularly useful as they can take account of the limited shortrange effects of fluctuations about the fixed rotation angles of the RIS model. This communication records the preliminary results of the application of WAXS procedures to the conformational analysis of poly(dimethylsiloxane) (PDMS) to determine the extent of the limitations of the RIS approach.

The conformation of PDMS in solution was studied in detail by Flory et al., ${ }^{13,14}$ who assigned three rotation states, trans, gauche, and - gauche, to each skeletal bond although recognising that the range of rotation angles available might be considerable because of the case of rotation about the $\mathrm{Si}-\mathrm{O}$ bond ${ }^{15,16}$ and the high chain flexibility which can perhaps be deduced from the particularly low glass transition temperature. ${ }^{17}$ More recently some configurational averages have been calculated for PDMS chains by Bruckner et al., ${ }^{9}$ in which the method of averaging takes account of all possible configurations. The conformational energy maps calculated in that work ${ }^{9}$ indicate that the assignment of three rotation states may be artificial, there being no distinct minima corresponding to the gauche or -gauche states. Thus PDMS seems a highly suitable polymer with which to test the utility of WAXS techniques for conformational studies of highly flexible polymers.

\section{EXPERIMENTAL}

The material used in this study was in the form of a lightly cross-linked sheet $(0.3 \mathrm{~mm}$ in thickness) of PDMS, the cross-linking agent being 2:4 dichlorobenzoyl peroxide. The Xray scattered intensities were measured using a symmetrical transmission diffractometer with an incident beam monochromator, ${ }^{18}$ over the range $2 \theta=3^{\circ}$ to $110^{\circ}$ in steps of $\Delta 2 \theta=0.2^{\circ}$,

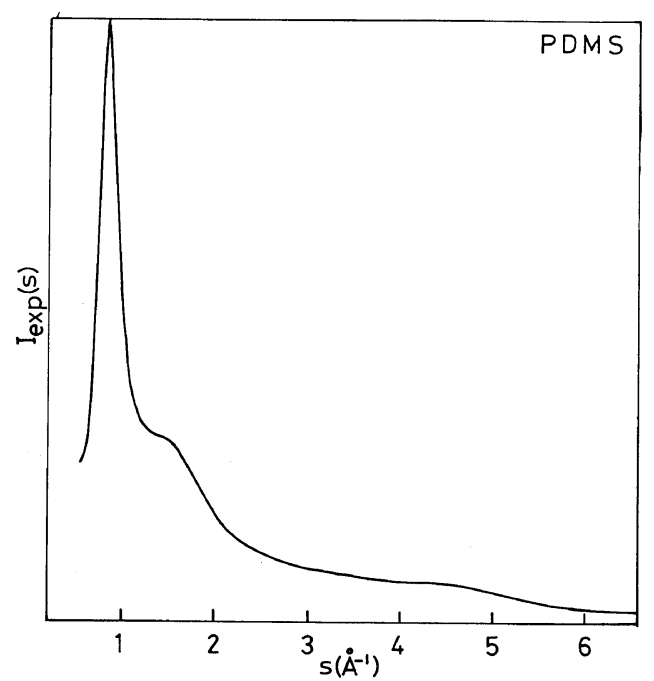

Figure 1. The intensity function $I(s)$ measured for poly(dimethylsiloxane) at $21^{\circ} \mathrm{C} . s=4 \pi \sin \theta / \lambda$, and the angle between incident and scattered paths is $2 \theta$. The vertical scale is in arbitrary units.

which corresponds with $\mathrm{Cu} K \alpha$ radiation to a scattering vector range of $s=0.21$ to $6.5 \AA^{-1}$ $(s=4 \pi \sin \theta / \lambda)$. The intensity values were smoothed using a cubic spline procedure ${ }^{19}$ and corrected for polarisation, absorption, multiple and incoherent scattering and normalised to electron units as described previously. ${ }^{18,20}$ The recorded intensity function $I_{\text {exp }}(s)$ is shown in Figure 1. There is an intense peak at $s \sim 0.85 \AA^{-1}$ which maximises towards the equator (i.e., normal to the extension axis) in scattering patterns obtained from oriented PDMS, ${ }^{21}$ and thus may be related to spatial correlations between chains segments. There are much weaker peaks at $s \sim 1.7 \AA^{-1}$ and $4.5 \AA^{-1}$. It has been found convenient for conformational studies to display the intensity data in the form of a $s$-weighted reduced intensity function $s i(s)$, in which the features are more evenly weighted over the $s$ range considered. The reduced intensity function may be obtained from the fully corrected intensity $I_{\text {corr }}(s)$ by:

$$
i(s)=k I_{\text {corr }}(s)-\sum_{i} c_{i} f_{i}^{2}(s)
$$




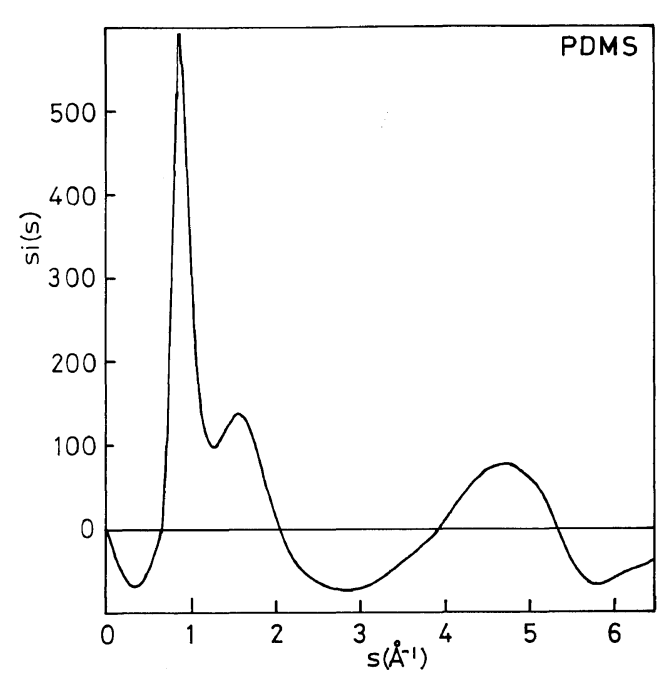

Figure 2. The $s$-weighted reduced intensity function $s i(s)$ for PDMS obtained from the experimental WAXS data of Figure 1. The vertical scale is (electrons) ${ }^{2} \AA^{-1}$

where $k$ is a normalisation factor and $\sum_{i} c_{i} f_{i}^{2}(s)$ is the independent scattering for one chemical repeat unit, where $f_{i}(s)$ is the atomic scattering factor $(i=\mathrm{C}, \mathrm{H}, \mathrm{Si}, \mathrm{O})$ and $c_{i}$ the number of the $i$-type atom. The $s i(s)$ function so obtained in this work is displayed in Figure 2. The scattering curve is different in some respects to those obtained from other non-crystalline polymers $^{12,20,22-25}$ for which there is an intense peak at $s \sim 1.5 \AA^{-1}$ and diffuse peaks at $s \sim 3$ and $5 \AA^{-1}$. The latter peaks may be associated with correlations within chain segments and hence their positions are related principally to carbon-carbon distances. It is therefore not suprising in view of the differences in bond lengths for $\mathrm{Si}-\mathrm{O}$ and $\mathrm{Si}-\mathrm{C}$ that these peaks are somewhat differently placed in the scattering curve for PDMS. The intense peak at $s \sim 0.85 \AA^{-1}$ is appreciably narrower than the corresponding peak in the curve for molten $\mathrm{PE}^{23}$ and natural rubber. ${ }^{24}$

\section{CONFORMATIONAL ANALYSIS}

The procedures used for the conformational analysis are similar to those employed in pre- vious WAXS studies of molten $\mathrm{PE},{ }^{23}$ polyphenylene sulphide, ${ }^{20}$ glassy polystyrene, ${ }^{25}$ and natural rubber. ${ }^{24}$ The limited level of spatial correlations between chain segments in noncrystalline polymeric materials reduces the extent of the scattering from such correlations to a first order peak at approximately $(2 \pi / d)$ where $d$ is the interaction distance between chain segments. ${ }^{23,26}$ Higher order peaks are suppressed by a combination of the spatial disorder and the spherical averaging of the molecular structure factor as a result of the orientational disorder. ${ }^{27}$ For such a system, the scattering at higher vectors, say $s>2(2 \pi / d)$ may be associated with the correlations within a single average chain segment. Thus for PDMS, the scattering for $s>2 \AA^{-1}$ may compared directly to the scattering calculated from sterically viable single chain models without the inclusion of interchain or intermolecular interactions. The geometrical parameters of the PDMS chain adopted in this study were the same as for previous studies, excepting the nature and distribution of the skeletal bond rotation angles. The procedures for calculating the scattering functions have been elaborated elsewhere, ${ }^{23,24}$ and calculations were performed for chains of 300 units, although only distances between atoms separated by less than 20 units were included. The latter device is to exclude correlations which are essentially interchain in nature. ${ }^{23}$ The scattering curves presented here are the results of averaging the si $(s)$ functions calculated for 10 such chains each of 300 units. The convergence of the averaged scattering function is quite rapid as the correlations it is based upon are shortrange in nature.

We shall consider first, the scattering calculated for models based upon the RIS approach, in which three rotation states were assigned (trans, gauche and -gauche). Their interdependence is modelled by two sets of statistical weights, ${ }^{13,14}$ one appropriate for the bonds about the Si atom $\left(U_{\mathrm{a}}\right)$ and the other for the bonds about $\mathrm{O}$ atom $\left(U_{\mathrm{b}}\right)$. 


$$
\begin{aligned}
& U_{\mathrm{a}}=\left[\begin{array}{ccc}
1 & \sigma & \sigma \\
1 & \sigma \psi & 0 \\
1 & 0 & \sigma \psi
\end{array}\right] \\
& U_{\mathrm{b}}=\left[\begin{array}{ccc}
1 & \sigma & \sigma \\
1 & \sigma & \sigma \omega \\
1 & \sigma \omega & \sigma
\end{array}\right]
\end{aligned}
$$

where $\sigma$ is related to the energy of the gauche state relative to trans $E_{\sigma}$ by $\exp \left(-E_{\sigma} / R T\right) . \psi$ and $\omega$ are parameters to take account of the additional energies of gauche following gauche of the same sign and gauche following gauche of the opposite sign respectively. Experimental values of various configuration dependent properties $^{28-31}$ are reproduced by letting $E_{\sigma}=$ $850, E_{\psi}=0$, and $E_{\omega}=1050 \mathrm{cal} \mathrm{mol}^{-1}$. Rotation angles corresponding to the trans, gauche, and - gauche states were 0,120 , and $-120^{\circ}$. The valence angles about the $\mathrm{Si}$ and $\mathrm{O}$ atoms differ by $\sim 33^{\circ}$ and thus the all-trans planar conformations leads to a closed ring, more extended configurations are generated by the inclusion of gauche isomers. Thus if $\sigma<1$ a positive temperature coefficient of the mean square end-to-end distance will result in accord with experimental measurements. ${ }^{14}$ Examination of the matrices (eq 2) indicates the strong influence that the value assigned to $E_{\sigma}$ will have upon the resultant configurations. Accordingly we retain this as a variable, the other parameters assigned the values given by Flory. ${ }^{13,14}$ In the procedures used in this work for the calculation of the scattering functions, the interatomic distances are derived from the atomic coordinates of a computer generated model, in which the assignment of the rotation states for a particular chain requires the conditional probabilities, which are easily evaluated from the statistical weights. ${ }^{24,32}$

Figure 3 shows the scattering calculated for random chains in which the distribution of rotation states was in accord with the conditional probabilities derived from the statisti-

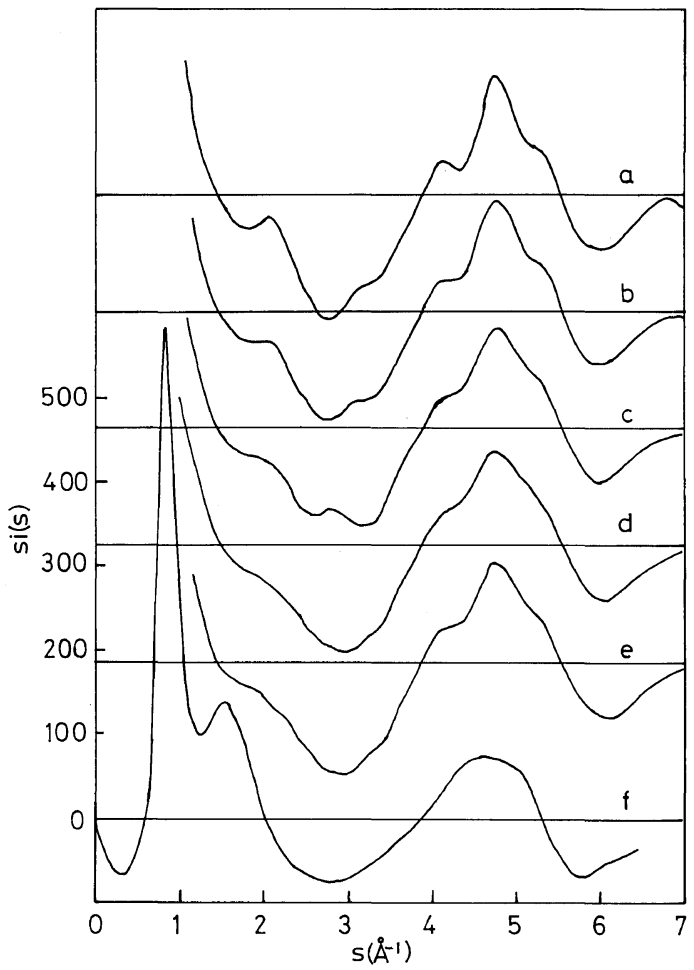

Figure 3. Reduced intensity functions si $(s)$ calculated for random model PDMS chains with the rotation states distributed using conditional probabilities derived from the statistical weight matrices defined in eq 2 , for a temperature of $21^{\circ} \mathrm{C}$ with the value of $E_{\sigma}$ set to a) 1000 , b) 750 , c) 500 , d) 250 , e) $0 \mathrm{cal} \mathrm{mol}^{-1}$ compared with f) the experimental function. The vertical scale marked is that for the experimental si(s) function and the units are (electron) $)^{2} \AA^{-1}$. The calculated $s i(s)$ curves are drawn at the same scale but the vertical scales are offset for convenience.

cal weights of eq 2 for particular values of $E_{\sigma}$. In view of the uncertainty in the exact extent of the interchain scattering, we shall principally consider the broad peak centered at $s \sim$ $4.5 \AA^{-1}$. The agreement between the experimental and calculated scattering functions is good in terms of the position of this broad peak, but all the calculated curves contain considerable detail not present in the experimental curve. There is some slight improvement in the curves for which $E_{\sigma}$ approaches $0 \mathrm{cal} \mathrm{mol}^{-1}$. For a chain with ill-defined minima in the conformational energy surface we 


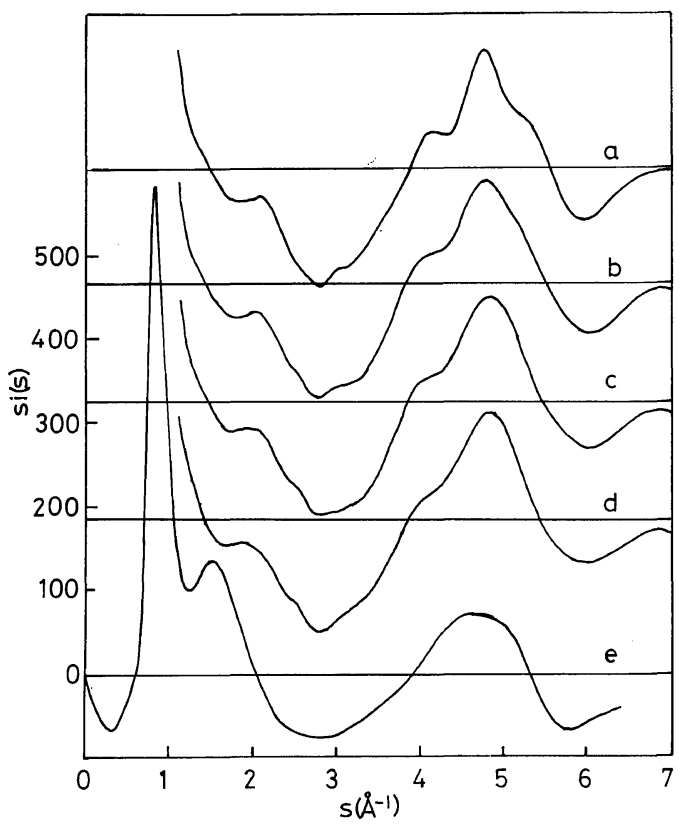

Figure 4. Reduced intensity functions $s i(s)$ calculated for random chains of PDMS with the rotation states distributed using conditional probabilities derived from the statistical weights of 2 for $T=21^{\circ} \mathrm{C}$ with the value of $E_{\sigma}$ set to $850 \mathrm{cal} \mathrm{mol}^{-1}$ as proposed by Flory et al. ${ }^{13}$ The angles assigned to the rotation states trans, gauche and - gauche are selected from Gaussian distributions centred at 0,120 and $-120^{\circ}$ with a standard deviation of a) 0 b) 10 c) 20 d) $30^{\circ}$. Curve a) corresponds to the RIS chain proposed by Flory et al. ${ }^{13}$ and curve e) is the experimental $s i(s)$ function. Vertical scale as in Figure 3.

would expect considerable fluctuations in the values of the bond rotation angles, and such distortions are included in the next model considered. We may easily incorporate random uncorrelated thermal-like fluctuations of the skeletal bond rotation angles into the scattering calculations, ${ }^{20,24}$ and we consider here Gaussian distributions of rotation angles centered about the rotation states, trans, gauche and -gauche. Figure 4 shows the calculated $s i(s)$ curves for such models compared with the experimental data. The scattering is derived for chains with $E_{\sigma}=850 \mathrm{cal} \mathrm{mol}^{-1}$, that is the value assigned by Flory et al. ${ }^{13,14}$ The scattering calculated for such a chain without thermal fluctuations (curve a in Figure 4) is in poor

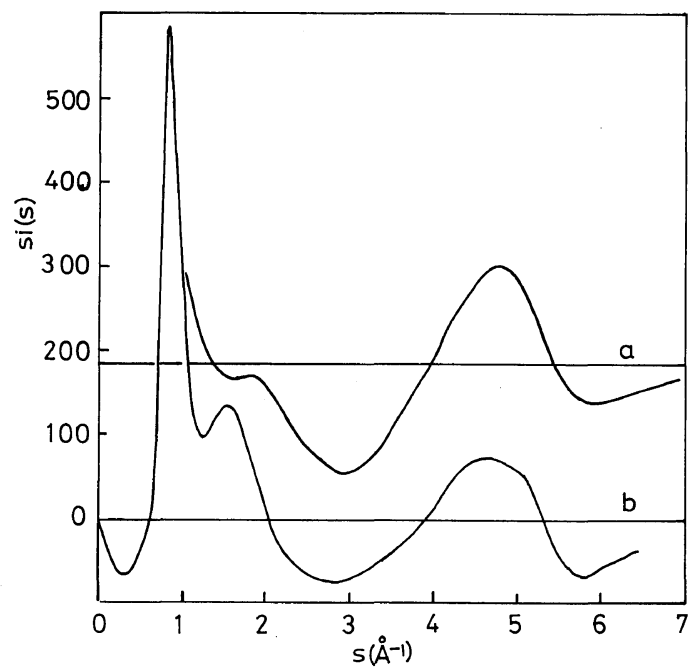

Figure 5. a) Reduced intensity function si(s) calculated for a random chain in which the skeletal bond rotation angles are independently selected at random from a uniform distribution 0 to $360^{\circ}$ compared to $\left.b\right)$ the experimental si(s) curve. Vertical scale as in Figure 3.

agreement with the experimental si $(s)$ curve as would be expected from comparisons made in Figure 3. The inclusion of thermal fluctuations has little effect when the standard deviation of the distribution is relatively small $\left(10\right.$ and $\left.20^{\circ}\right)$ as was noted in a previous study of molten $\mathrm{PE}^{23}$ Large fluctuations with a standard deviation of $30^{\circ}$ introduce some improvement, however fluctuations of the magnitude, and particularly those which are uncorrelated with those of neighbouring bonds indicate considerable delocalisation of the rotation states. Figures 3 and 4 show that neither adjustment of the parameters of the RIS chain nor the introduction of thermal-like fluctuations leads to a satisfactory comparison between calculated and experimental scattering functions. It is clear that the application of the RIS model which successfully represented the mean square end-to-end vector and its temperature coefficient ${ }^{13,14}$ is an inadequate description of the local conformation. Figure 5 shows the scattering calculated from a model chain in which the bond rotation angles can adopt any value independent of the neighbouring bonds. 
The agreement in the scattering vector range $s>2.5 \AA^{-1}$ is marked. The descrepancies at lower scattering vectors are clearly the result of the complications of interchain correlations. Obviously, such a chain configuration will have some sterically unacceptable conformations, although if we consider the energy map presented by Bruckner et al. ${ }^{9}$ or even simple conformational maps indicating Van der Waals type overlaps, ${ }^{33}$ we may see that there are relatively few conformations which have a prohibitively high energy. In fact only those in the region of the cis-cis type may be totally excluded. However, Flory et al. ${ }^{13}$ found that a chain with independent rotations of the skeletal bonds provided chain configurational properties at variance with experiment. We do not propose at this juncture to refine the local conformational model since it is clear that such a refinement must also include the comparison of larger distance correlations and such work is in hand. However, we may conclude that there is no evidence to support a local conformational model for PDMS in which the skeletal bond rotation angles are restricted to three values, and that the WAXS scattering analysis suggests considerable freedom of bond rotation in accord with spectroscopic data ${ }^{15,16}$ and the physical properties of PDMS.

\section{DISCUSSION}

To a certain extent, concern for the reliability of models in reproducing observable properties, is related to the use to which the model will be extended. Thus for configurational properties of flexible polymers in which fluctuations of the skeletal bond rotations angles probably have a self-cancelling effect, ${ }^{11,34}$ the RIS model may provide an adequate description. However, there may be serious errors if that model is used to predict the behaviour of the material in situations where such behaviour is dependent upon local interactions, as for example in dynamic prop- erties $^{35}$ and perhaps in deformation processes. Naturally the most exacting test of the correctness of any model is compare all of the properties that may be calculated with experimental observations, which is clearly a daunting task. However, a comparison of the spatial distribution function or its Fourier transform over all values of $r$ or the scattering vector in the case of its transform offers a reasonable compromise. This could be achieved by a combination of SANS and WAXS experiments, although the scattering vector range of $s \sim 0.8$ to $2.0 \AA^{-1}$ is larger inaccessible because of interchain interactions. It is suggested that WAXS techniques could be particularly useful in resolving some of the detail of the local conformation, as has been achieved for example, in establishing the plurality of the rotation states for molten $\mathrm{PE}^{23}$ and determining the valence angles in poly(methylmethacrylate). ${ }^{11,18,36}$

Acknowledgements. One of the authors (GRM) gratefully acknowledges the support, via a Fellowship, of the Japan Society for the Promotion of Science, and the hospitality of Professor Odajima during the period spent at Hokkaido University. The authors thank Claude Hepburn of Loughborough University U. K. for providing the sample of PDMS.

\section{REFERENCES}

1. P. J. Flory, "Statistical Mechanics of Chain Molecules," Interscience, New York, 1969.

2. M. V. Volkenstein, "Configurational Statistics of Polymetric Chains," Interscience, New York, 1969.

3. R. Cook and M. Moon, Macromolecules, 11, 1054 (1978).

4. R. Cook and M. Moon, Macromolecules, 13, 1537 (1980).

5. T. Oyama and K. Shiokawa, Polym. J., 13, 1145 (1981).

6. G. Allegra and A. Immirzi, Makromol. Chemie, 124, 70 (1969).

7. G. Allegra, M. Calligaris, and L. Ranaccio, Macromolecules, 6, 390, 397 (1973).

8. G. Allegra and S. Bruckner, Macromolecules, 10, 106 (1977). 
9. S. Bruckner and L. Malpezzi, Makromol. Chem., 183, 2033 (1982).

10. M. L. Mansfield, J. Chem. Phys., 72, 3923 (1980).

11. M. L. Mansfield, Macromolecules, 16, 1863 (1983).

12. R. Lovell, G. R. Mitchell, and A. H. Windle, Faraday Discuss., 68, 46 (1979).

13. P. J. Flory, V. Crescenzi, and J. E. Mark, J. Am. Chem. Soc., 86, 146 (1964).

14. ref. $1, \mathrm{p} 174$.

15. D. W. Scott, J. F. Messerly, S. S. Todd, G. B. Guthrie, I. A. Hossenlopp, R. T. Moore, A. Osborn, W. T. Berg, and J. P. McGullough, J. Phys. Chem., 65, 1320 (1961).

16. C. D. LeCroix, R. F. Curl, P. M. Mckinney, and R. J. Meyers, J. Mol. Spectrosc., 53, 250 (1974).

17. "Polymer Handbook," J. Brandrup and E. H. Immergut, Wiley Interscience, New York, 1975.

18. G. R. Mitchell and A. H. Windle, Colloid Polym. Sci., 260, 754 (1982).

19. M. Dixon, A. C. Wright, and P. Hutchinson, Nucl. Instrumental Methods, 143, 379 (1977).

20. T. P. H. Jones, G. R. Mitchell, and A. H. Windle, Colloid Polym. Sci., 261, 110 (1983).

21. S. M. Ohlberg, L. E. Alexander, and E. L. Warrick,
J. Polym. Sci., 27, 1 (1958).

22. N. Numakawa and A. Odajima, Polym. J., 13, 879 (1982).

23. G. R. Mitchell, R. Lovell, and A. H. Windle, Polymer, 23, 1273 (1982).

24. G. R. Mitchell, to be submitted to Colloid Polym. Sci.

25. G. R. Mitchell and A. H. Windle, Polymer, in press.

26. G. R. Mitchell and R. Lovell, and A. H. Windle, Polymer, 21, 989 (1980).

27. G. R. Mitchell and A. Odajima, in preparation.

28. P. J. Flory and J. A. Semlyen, J. Am. Chem. Soc., 88, 3209 (1966).

29. M. H. Liberman, Y. Abe, and P. J. Flory, Macromolecules, 5, 550 (1972).

30. J. E. Mark, J. Chem. Phys., 49, 1398 (1968).

31. W. L. Mattice, Macromolecules, 11, 517 (1978).

32. ref. $1, \mathrm{p} 74$.

33. G. R. Mitchell, unpublished work.

34. ref. $1, \mathrm{p} 57$.

35. E. Helfand, Z. Wasserman, and T. A. Weber, $J$. Phys. Chem., 70, 2016 (1979).

36. R. Lovell and A. H. Windle, Polymer, 22, 175 (1981). 